\title{
Research on Laser Dazzle Simulation Using ZEMAX Software and MATLAB
}

\author{
Niu jin ${ }^{1,2, *}$, Xu xiping ${ }^{1}$, and An zhiyong ${ }^{1}$ \\ ${ }^{1}$ School of Optoelectronic Engineering, Changchun University of Science and Technology, China \\ ${ }^{2}$ Weaponry Equipment Research Institute of China Ordnance Equipment Group, China
}

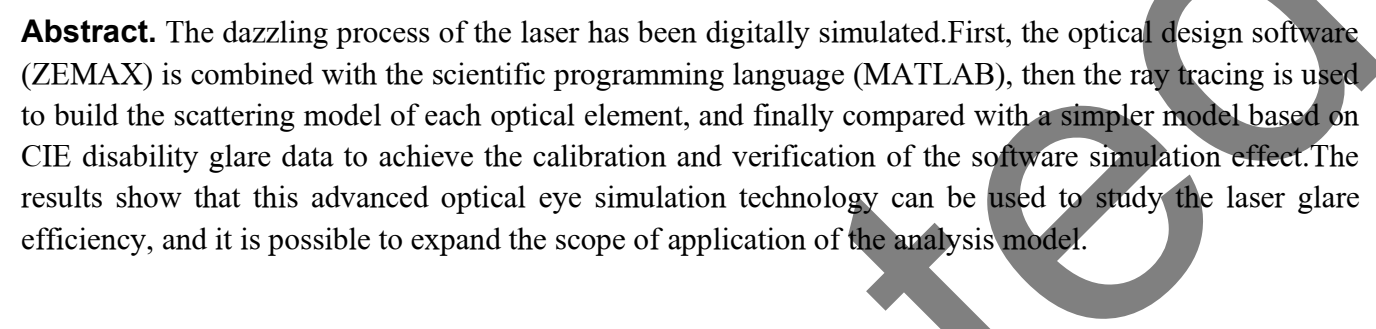

\section{INTRODUCTION}

Laser dazzle gained public awareness due to the increase in availability of visible wavelength lasers with powers well above laser pointers traditionally used presentations. It has been very popular in the civil field $[1,2]$.

The standard International Commission on Illumination (CIE) equation is a collection of research and development results of different researchers [3, 4], and has been calibrated by McLin et al. [5] for laser glare performance and integrated by Williamson. A visual tool for blinding performance research. These models can accurately simulate the dazzling effect in different situations, but they are not suitable for studying the addition of different optical components or simulating a specific real scene containing all the contributing parameters.

\section{Laser dazzling simulation modeling}

The human eye is one of the most complex organs of the human body. Although optically it can be considered a simple refractive system, the way the different components behave as different objects are observed, at different distances, under different illumination, and how the elements change with aging(and from person to person) poses real challenges to researchers.

\subsection{Ray tracing modeling for simulating human eye design}

A typical optical model is implemented using an optical design program with continuous ray tracing. That is, trace light from one surface to another in a sequential manner. Although this method can use fast and accurate tools to design and analyze optical systems, when simulating the human eye, sequential ray tracing does not fully consider an important phenomenon: scattering. To account for scattering, a common method is to use non-sequential ray tracing. This technology is based on the Monte Carlo method, which allows automatic light splitting to allow light to contact the surface in any order and number of times. Using non-sequential ray tracing, you can also define light sources and detectors in a more practical way, and users are not limited to the sequential placement of elements. However, both methods have advantages and disadvantages, and the specific choice depends on the overall analysis and research [6].

Generally, eye models implemented in optical design programs are "static"; that is, they do not allow dynamic interaction with users. Therefore, the most commonly used optical design software package (ZEMAX) and the popular scientific programming tool (MATLAB) are used after information exchange. Its main input items include the age of the human eye, the pupil size and the distance to the observed object, and also consider the scattering parameters of the main contributors (corneal, lens and fundus). Using this data, a set of parameters that define the eye can be obtained, and the ZEMAX eye design is created.

The human eye simulation module has two interfaces for entering/changing relevant parameters during the design process. As shown in Fig. 1, the human eye simulation module is responsible for defining the optical design of the human eye based on the designer's input. In its start interface, the subject's age, pupil size and distance from the object to be observed are defined. The second interface (parameterized interface) allows designers to change these parameters when necessary (for example, to add gradient index properties to the lens, consider eccentric concave positions, and even apply parameters of other eye models that they think are more

\footnotetext{
* Corresponding author: niujin0615@163.com
} 
appropriate). From this interface, the designer can also start the optical design of the eye by entering the automatically created ZEMAX file or advancing to a specific module that can be developed using the same principle to analyze different phenomena related to optical simulation.

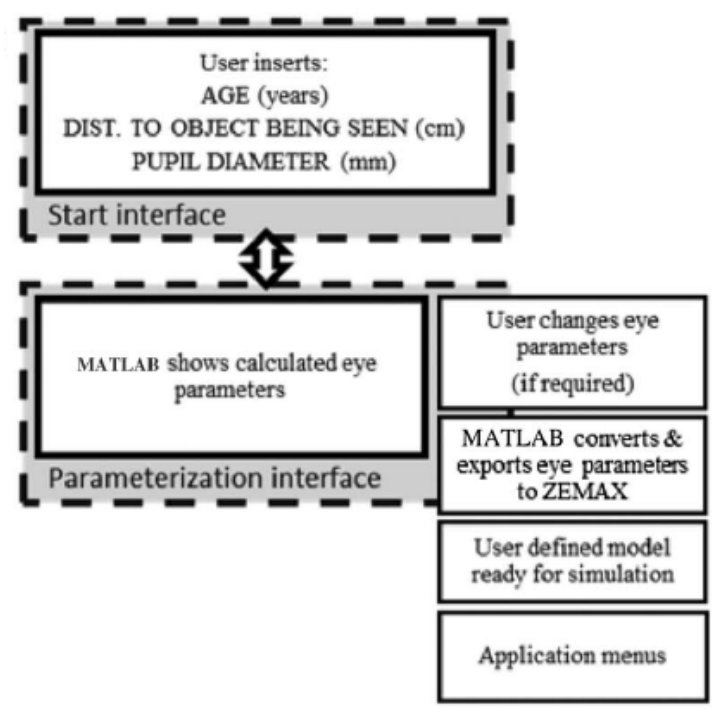

Fig. 1. Human eye simulation model flow chart

\subsection{Laser dazzling scene simulation modeling}

From the content of 2.1, you can get a set of parameters that define the eyes, and created the ZEMAX eye design. In order to simulate the real laser glare effect, an image model for superimposing the laser pattern on the digital image was also established.

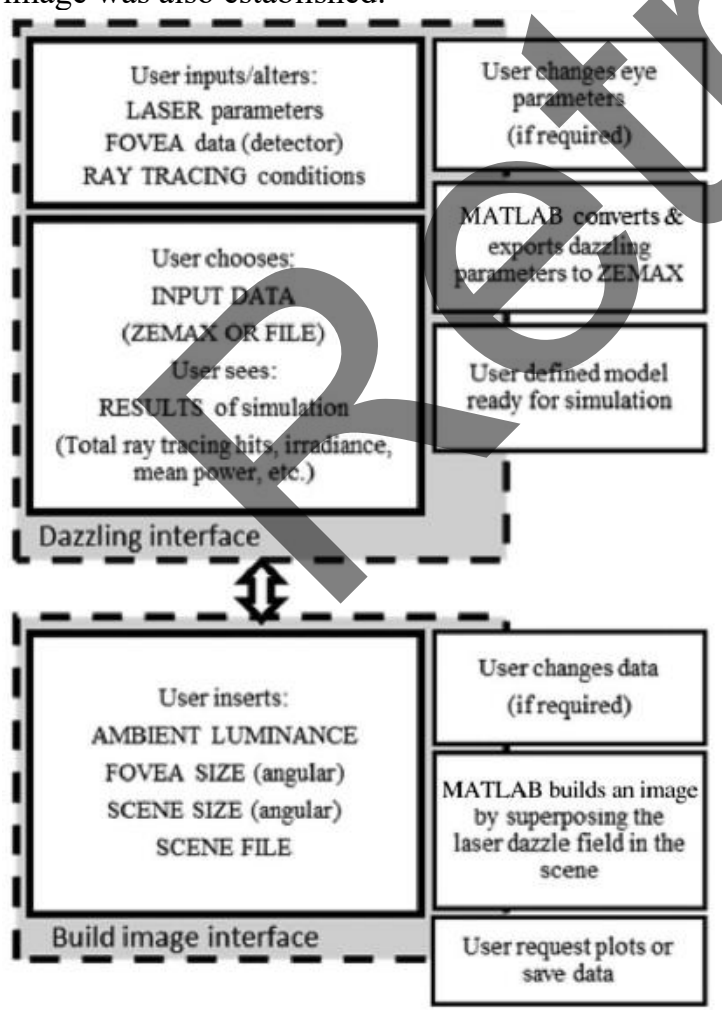

Fig. 2. Laser Dazzling Image Model flow chart

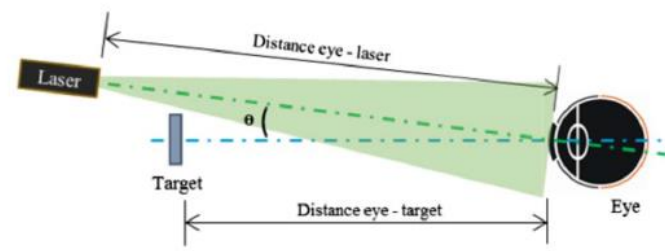

Fig.3. Schematic illustrating a laser dazzle scenario

Fig.2 illustrates the process of the laser dazzling image model, Fig.3 shows a simplified schematic diagram of the laser dazzling scheme, and Table $\mathbf{1}$ lists the necessary inputs to define this scheme.In the first interface of the laser glare module (the glare interface), the user can enter/change the laser parameters, the characteristics of the detection element in the retina (imaging area) and the ray tracing conditions. Designers can also import data from previous simulations (or save the current simulation). The program then allows the user to export all parameters to ZEMAX and perform ray tracing in it. Using the data obtained after ray tracing, the MATLABZEMAX toolbox was used to obtain an irradiance matrix (a table of illuminance values for the entire retina) and give a set of results.

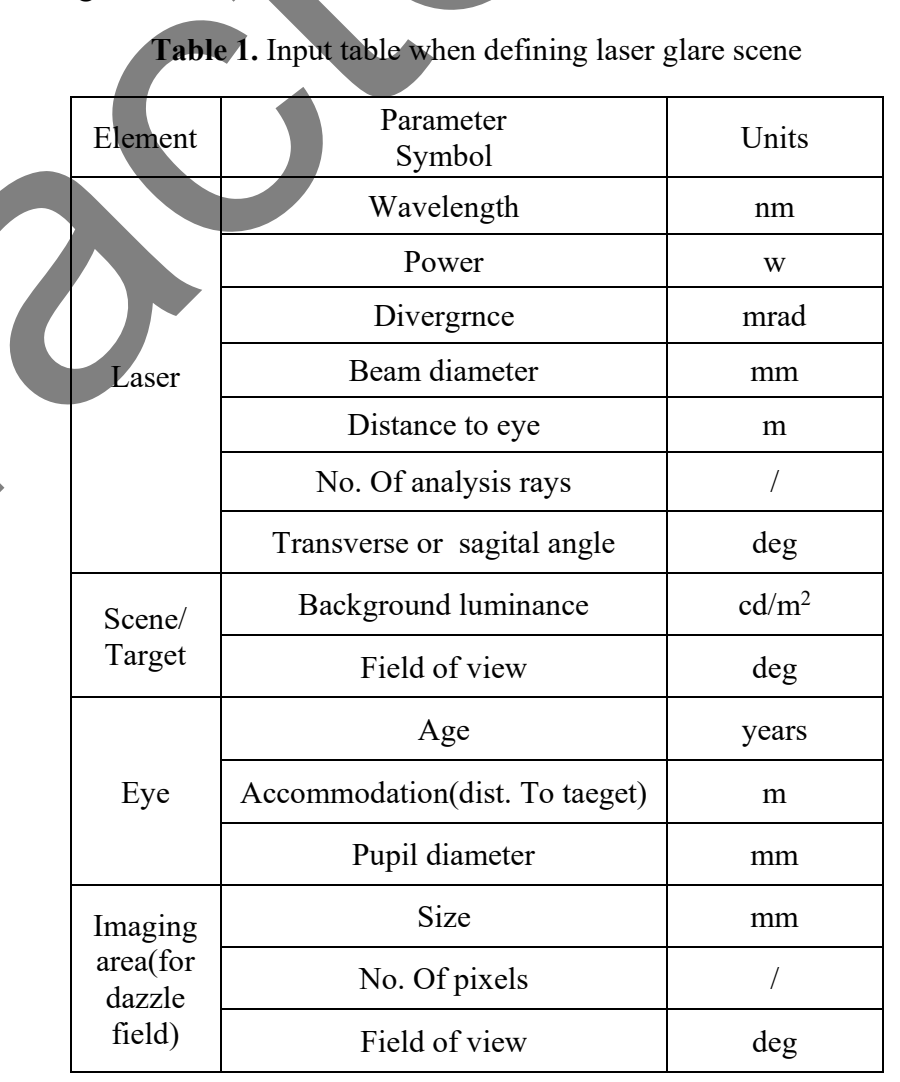

With the data obtained, the next task is to build a graph to load the image and overlay the laser pattern. When we input/change the ambient brightness of the scene, the FoV (angle size) of the detector and scene, and the scene file, and use the irradiance matrix from the simulation to create a laser glare image, as shown in Figure 4. 


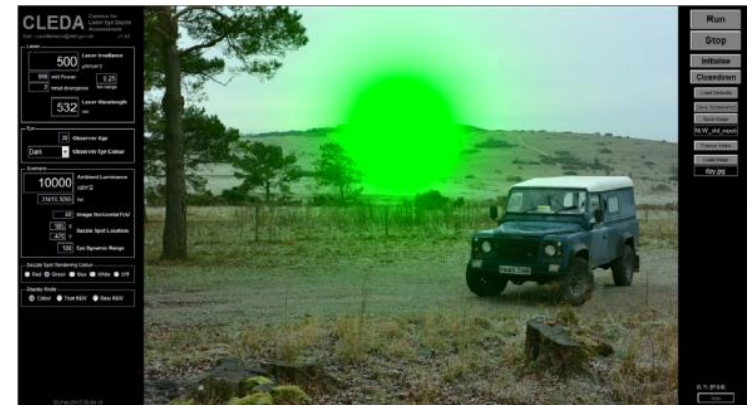

Fig. 4. Laser dazzling image overlay rendering

Convert the image irradiance to brightness, and then create an 8-bit pixel level from 0 (black) to 255 (saturated) dazzling outline, you can add the image to the scene; the saturation of the image is determined by the instantaneous dynamic range of the eye, That is, when the ratio of laser pixel brightness to ambient brightness is equal to or greater than this dynamic range, the pixel is considered saturated; if the ratio is below the reciprocal of the eye range, the pixel does not contribute anything to the final image. By assigning a value of (ratio) $\times(255$ / eye range) to these pixels, a smooth transition can be achieved between these two levels.

\section{Establishment of human scattering simulation model}

Contrary to the commonly used parameters of the eye (curvature, distance, etc.), the scattering data on the various components of the human eye are few and incomplete. Nevertheless, this article takes a lot of empirical values to use the human eye scattering data introduced by MATLAB routines, and will analyze the scattering data of each eye element.

Massive scattering of optical elements in ZEMAX is a phenomenon that requires the use of its discontinuous mode. Two models already provided in ZEMAX can be used: Rayleigh and Henyey - Greenstein. The premise of using these two models is that the scattering length (the reciprocal of the absorption coefficient in millimeters) and the transmittance of the medium must be specified (to simulate the loss due to absorption in each scattering event). For Rayleigh scattering, the reference wavelength should be added, and the HenyeyGreenstein model requires the anisotropic coefficient $g$. The latter represents the average cosine of the scattering angle and varies between -1 and 1 . For $g>0$, forward scattering dominates, and for $\mathrm{g}<0$, backscattering dominates.

\subsection{Cornea}

Although it can be considered that the first element of the eye (the cornea) is highly transparent, transmission loss must be considered, such as loss due to scattering effects.
The initial transmission loss is due to reflection at the cornea-air interface. Because the refractive index of the cornea is considered to be about 1.377 , the reflectivity at this interface can be assumed to be $2.5 \%$, although for light with a higher angle of incidence, the reflectivity can be increased to $5 \%$. At the cornea-water interface, the reflectivity is 2 orders of magnitude smaller.

Several experimental measurements have been reported regarding transmission. Two measurements can be considered: direct transmission and total transmission. The latter considers the overall transmittance, combining diffuse and collimated light, while direct transmission regards only collimated light (small angle transmission). In 1994, van den Berg and Tan [7] collected a set of experimental data from several authors and created a model for corneal spectral transmittance:

$$
\mathrm{T}(\lambda)=10^{-0.016-\mathrm{c} \lambda^{-4}}
$$

where c represents a constant depending on the type of measurement being considered, and the wavelength, $\lambda$, is in $\mathrm{nm}$. If the acceptance (full) angle of the detecting element is $\sim 1$ deg (direct transmission), then

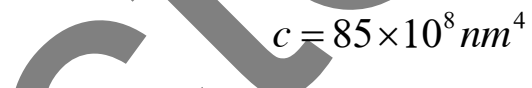

while if that angle is $\sim 180 \mathrm{deg}$ (total transmission), then

$$
c=21 \times 10^{8} \mathrm{~nm}^{4}
$$

Figure 5 shows the resulting transmittance values. Also important are the observations that, in general, the shape of the spectral transmission seems independent of age .

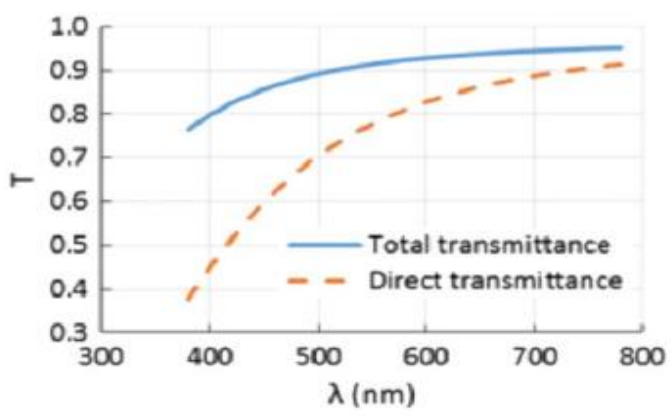

Flg. 5. Corneal spectral transmittance as calculated using Eq. (1)

Besides knowing the degree of transparency of the cornea, the simulation of light propagation through this element requires knowledge of the scattering coefficient, $\alpha$ s (considering that scattering is the main mechanism involved). The relationship between transmittance and scattering (e.g., [8]) is given by

$$
T_{s}(z)=\exp \left(-\alpha_{s} z\right)
$$

where $\alpha_{s}$ is the product of the cross section of the scatterers, $\sigma_{\mathrm{s}}$, and their density, $\rho$, that is, $\alpha_{\mathrm{s}}=\sigma_{\mathrm{s}} \rho_{\mathrm{s}}$. 
If several scatterers are present, the overall scattering coefficient is obtained by summing the different scattering coefficients.

Although the cornea's composition is complex, most models for light transmission through the human cornea consider a monodisperse system of fibrils (long dielectric nonabsorbing cylinders) $[9,10]$. Under this assumption, Doutch et al. developed a model based on knowledge of fibrils characteristics measured by OCT, $\mathrm{x}$-ray diffraction, and electron micrographs. Based on their formulas, and considering just the cornea's central position, we obtained the spectral variation of the expected scattering coefficient (Fig. 6).

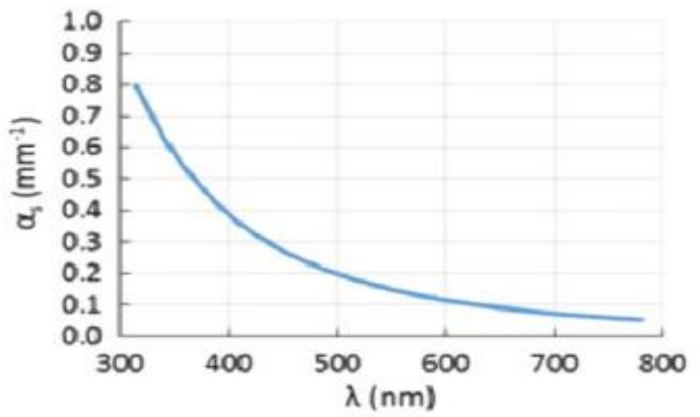

Fig. 6. Spectral variation of the cornea's scattering coefficients and length, based on the model developed by Doutch et al. [10].

In our simulator, the necessary parameters are the scattering length and the transmittance. The latter is used to simulate losses due to absorption in each scattering event. To test this implementation, we simulated only the cornea (and the aqueous humor) irradiated by a monochromatic (single wavelength), collimated, source of light with $1 \mathrm{~W}$ power and $2 \mathrm{~mm}$ beam diameter. We traced $5 \times 107$ rays (having a higher number did not change the data) and measured the power at the exit of the cornea with a $750 \times 750$ pixel detector.

Fig. 7 compares the transmittance obtained from the different models, including the implementation in ZEMAX (dEYEnamic simulator). The difference between the data from Doutch et al. [29] and that of our implementation is about $3 \%$, which is acceptable considering that the cornea's thicknesses are different (ours is $24 \mu \mathrm{m}$ thicker); in reality, this value is not constant as it increases from the center to the periphery (a phenomenon we did not consider). Nevertheless, our results are in accordance with the experimental average transmission presented by this model (about $2.5 \%$ less transmittance at $500 \mathrm{~nm}$ ) and within the error stated for their data $(2 \%)$.

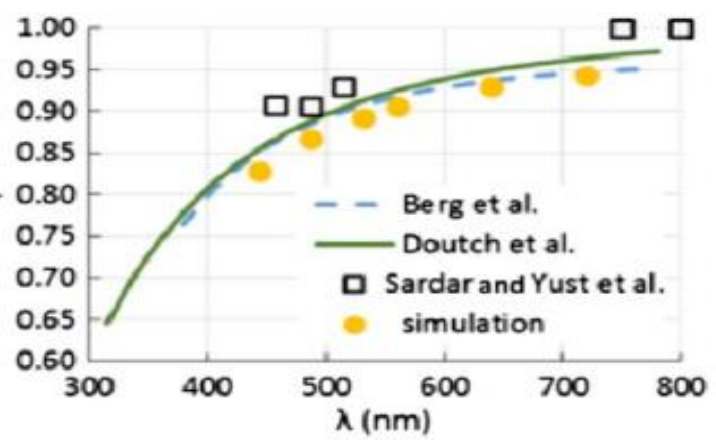

Flg.7.Simulation data of human cornea total transmittance[1116]

\section{2 lens}

In addition to being dependent on the wavelength of light, the optical parameters of the human eye lens also depend on age and other factors. However, from these studies, it is possible to determine the spectral transmittance values of lenses of different ages based on the entire data set.

We derived a function to describe the increase of optical density $\left(\log D=1 / T_{\alpha}\right)$ with age, based on Moreland's data. This function allows the optical density of an average observer between the ages of 20 and 60 years to be estimated using

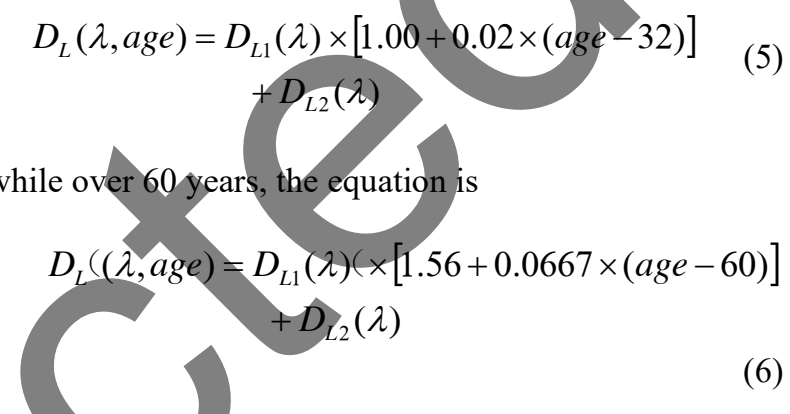

where $D_{\mathrm{L} 1}$ and $\mathrm{D}_{\mathrm{L} 2}$ are two optical density contributions, not varying with age (Fig.7). The latter only contributes for wavelengths lower or equal to $450 \mathrm{~nm}$.

The more recent study of Artigas et al.[16] reported the transmittance of in vitro lenses obtained from eyes used for corneal transplants, without presenting any pathology. From their observations and in accordance with several other studies, the variation in transmission with age is only significant for elderly eyes. Above 60 years old, transmission decreases according to

$$
T^{\prime} \text { (age) }=-2.3452 \times 10^{-2} \text { age }+2.3445
$$

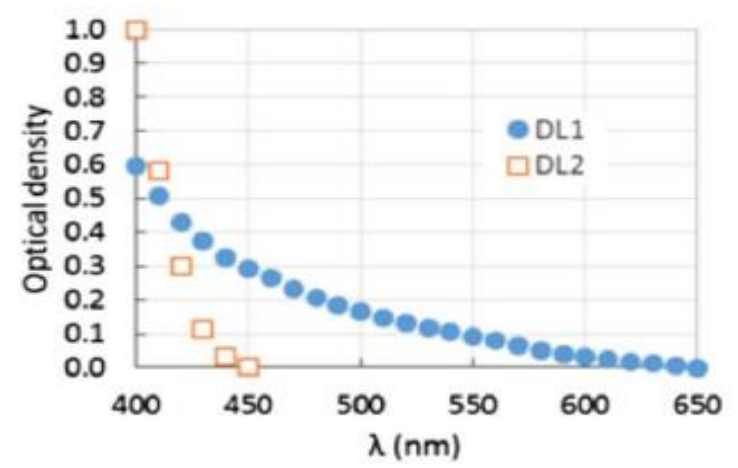

Flg.8. Optical density data contributing for Eqs.(5) and (6)

Depending on the age range being considered, we include the spectral variation by fitting data to define the following equations:

For age $\geqslant 60$, 


$$
T(\lambda, \text { age })=0.5\left[1+\operatorname{erf}\left(\frac{\lambda-405}{63 \sqrt{2}}-1.05\right)\right] T^{\prime}(\text { age })(8)
$$$$
\text { For } 60<\text { age } \leqslant 40 \text {, }
$$

$$
T(\lambda, \text { age })=T(\lambda)=0.5\left[1+\operatorname{erf}\left(\frac{\lambda-470}{45 \sqrt{2}}\right)\right]
$$

And,for age $<40$,

$$
T(\lambda, \text { age })=T(\lambda)=0.5\left[1+\operatorname{erf}\left(\frac{\lambda-\mu}{\sigma \sqrt{2}}\right)\right]
$$

where $\mu=370 \mathrm{~nm}$ and $\sigma=50 \mathrm{~nm}$ if $\lambda>400 \mathrm{~nm}$, otherwise $\mu=402 \mathrm{~nm}$ and $\sigma=10 \mathrm{~nm}$. The wavelength in these formulas is in $\mathrm{nm}$. As before, the calculated results should be considered only as an estimate. To illustrate, according to [16], the standard deviation for adult subjects (younger than 60 years old) resides between $6 \%$ and $16 \%$ and for elderly subjects (older than 60 years old) between $6 \%$ and $21 \%$.

\section{CONCLUSIONS}

The increasing number of laser dazzle events has motivated the study of glare impacts on the eye. CIE models for disability glare already quite accurately represent dazzle effects on the eye.

However, these models are based on analytical equations and have some limitations when representing the impact of the different parameters of the eye on the phenomena. Also, if one wants to develop and/or analyze the performance under laser dazzle of optical systems, they are not suitable for the task.

In order to contribute to the development of a different kind of laser dazzle simulator, we developed an optical simulator of the human eye (dEYEnamic simulator), combining the use of optical design software (ZEMAX) with a scientific programing language (MATLAB) through a dedicated toolbox. The developed interface allows the user to implement and analyze aparticular dazzle scenario using effective field parameters such as the distances from the laser to the eye, the level of accommodation of the eye (by indicating the distance to a particular object being observed), laser divergence, beam size, etc. To accomplish this, an existing optical model of the eye was upgraded with scattering data found in the literature.

Contrary to usual glare analysis, our model requires knowledge of the scattering model and parameters of each specific element of the human eye. The data are somehow scarce and,as is common with biological data, presents a high dispersion of values. This leads us to present a detailed review of the state of the art on the knowledge of these parameters and, as far as possible with the existing literature, to validate its implementation in ZEMAX.

\section{References}

1. C. A. Williamson and L. N. McLin, "Nominal ocular dazzle distance (NOOD)," Appl. Opt. 54, 1564-1572 (2015).

2. LaserPointerSafety.com, "US: FAA-reported laser incidents up significantly in 1st half of 2015," July2015

3. CIE Equations for Disability Glare Vienna: Commission Internationale de I'Eclairage (CIE, 2002).

4. J. J. Vos, "On the cause of disability glare and its dependence on glare angle, age and ocular pigmentation," Clin. Exp. Optom. 86, 363-370 (2003).

5. L. N. McLin, P. A. Smith, L. E. Barnes, J. R. Dykes, T. Kuyk, B. J. Novar, P. V. Garcia, and C. A. Williamson, "Scaling laser disability glare functions with ' $\mathrm{k}$ ' factors to predict dazzle," in International Laser Safety Conference, Orlando, USA, 21 March 2013 (Laser Institute of America, 2013).

6. M. Stevenson, "Optical software: which program is right for me?" Opt. Laser Eur. 141, 29-32 (2006).

7. T. van den Berg and K. Tan, "Light transmittance of the human cornea from 320 to $700 \mathrm{~nm}$ for different ages," Vis. Res. 34(11), 1453-1456 (1994).

8. M.H.Niemz,Laser-Tissue Interactions:Fundamentals and Applications (Springer, 2007).

9. V. Tuchin, "Light scattering study of tissues," Phys.-Uspekhi 40, 495-515 (1997).

10. J. Doutch, A. Quantock, V. Smith, and K. Meek, "Light transmission in the human cornea as a function of position across the ocular surface: theoretical and experimental aspects," Biophys. J. 95, 5092-5099 (2008).

11. T. van den Berg and K. Tan, "Light transmittance of the human cornea from 320 to $700 \mathrm{~nm}$ for different ages," Vis. Res. 34(11), 1453-1456 (1994).

12. M. H. Niemz, Laser-Tissue Interactions: Fundamentals and Applications (Springer, 2007).

13. D. Sardar, B. Yust, F. Barrera, L. Mimun, and A. Tsin, "Optical absorption and scattering of bovine cornea, lens and retina in the visible region," Lasers Med. Sci. 24, 839-847 (2009).

14. D. Sardar, R. Yow, G. Swanland, R. Thomas, and A. Tsin, Optical Properties of Ocular Tissues in the Near Infrared Region, HumanEffectiveness Directorate, Directed Energy Bioeffects Division (Air Force Research Laboratory, 2005).

15. B.Yust,L.Mimun, and D. Sardar, "Optical absorption and scattering of bovine cornea, lens, and retina in the near-infrared region," Lasers Med. Sci. 27, 413422 (2012).

16. J. Artigas, A. Filipe, A. Navea, A. Fandião, and C. Artigas, "Spectral transmission of the human crystalline lens in adult and elderly persons: color and total transmission of visible light," Invest. Ophtalmol. Visual Sci. 53, 4076-4084 (2012). 